\title{
ENHANCED ENERGY CONVERSION AS A RESULT OF FLUID-SOLID INTERACTION IN MICRO- AND NANOSCALE
}

\author{
Janusz Badur, Pawe€ ZióŁkowski, Sebastian Kornet, Tomasz Kowalczyk, \\ Kamil BANAŚ, Mateusz BryK \\ Institute of Fluid Flow Machinery, Energy Conversion Department, Polish Academy of Sciences, Gdańsk, Poland \\ Piotr JózeF ZióŁKOWski \\ Institute of Fluid Flow Machinery, Energy Conversion Department, Polish Academy of Sciences, Gdańsk, Poland \\ Gdańsk University of Technology, Faculty of Civil and Environmental Engineering, Gdańsk, Poland \\ e-mail: pziolkowski@imp.gda.pl
}

Michaє StajnKe

Institute of Fluid Flow Machinery, Energy Conversion Department, Polish Academy of Sciences, Gdańsk, Poland

\begin{abstract}
It is known that nano- and micromechanics require new approaches to right describing of surface-like phenomena which lead to an enhanced energy conversion. In this work, a general form of surface forces that consist of a contribution from both the friction and mobility components has been extended to collect the effects of bulk and surface motion of a fluid. Quite similar impact can be observed for a solid-fluid mixture, where the principle of effective stress for this new type of approach should be considered from the very beginning. The second motivation of our work is to present the multiscale domain of fluid-solid interaction which describes some "emergence effects" for materials with especially high volumetric surface densities.
\end{abstract}

Keywords: enhanced energy conversion, micro- and nanomechanics, fluid-solid interaction, pressure and thermally driven flow

\section{Introduction}

Though the classical continuous mechanics theory is enormously useful, it does not take into account all the phenomena that occur in the fluid-solid interface at the micro- and nanoscale. Thus, new approaches in continuum thermodynamics with slip velocity, thermal transpiration and a variety of different "jump" phenomena on the surface like: temperature, concentration, pressure, electric potential, order parameter, etc., are developed with comparison to experimental results (Badur et al., 2011, 2015; Henry and Minier, 2014; Kowalewski et al., 2016; Lewandowski et al., 2011; Nakielski et al., 2015; Nitoń et al., 2013; O'Hare et al., 2007; Thomson and Trojan, 1997; Ziółkowski and Badur, 2014). Moreover, these surface processes, in general, lead to some enhancement of energy conversion in comparison to the bulk classical transport model (Badur et al., 2015; Lemański and Karcz, 2008; Morini et al., 2011; Nakielski et al., 2015). A similar impact can be observed for porous saturated solids, when the Terzaghi principle of effective stress leads to a new situation in which the exchange of momentum and thermal energy in such continua undergo in a more complex manner (Badur et al., 2011, 2015; Lemański and Karcz, 2008; Thomson and Trojan, 1997; Ziółkowski and Badur, 2014). The enhanced conversion of energy is observed in new micro- and nanodevices as outside devices which work in power plants.

Practically, we should use the surface phenomena to improve the exchange of mass, momentum and energy (Kucaba-Piętal et al., 2009; Lemański and Karcz, 2008; Lewandowski et al., 
2011; Morini et al., 2011; O'Hare et al., 2007; Reese et al., 2003; Thomson and Trojan, 1997; Ziółkowski and Badur, 2014). Also quite new challenges for accurate prediction and assessment of the material effort (the risk of fracture) are developed for these processes (Banaszkiewicz, 2015; Banaszkiewicz and Rehmus-Forc, 2015; Pęcherski et al., 2011; Vadillo et al., 2011). A schematic presentation of these emergencing asspects of the modelling of fluid-solid interactions in the multiscale domain is highlighted in Fig. 1.

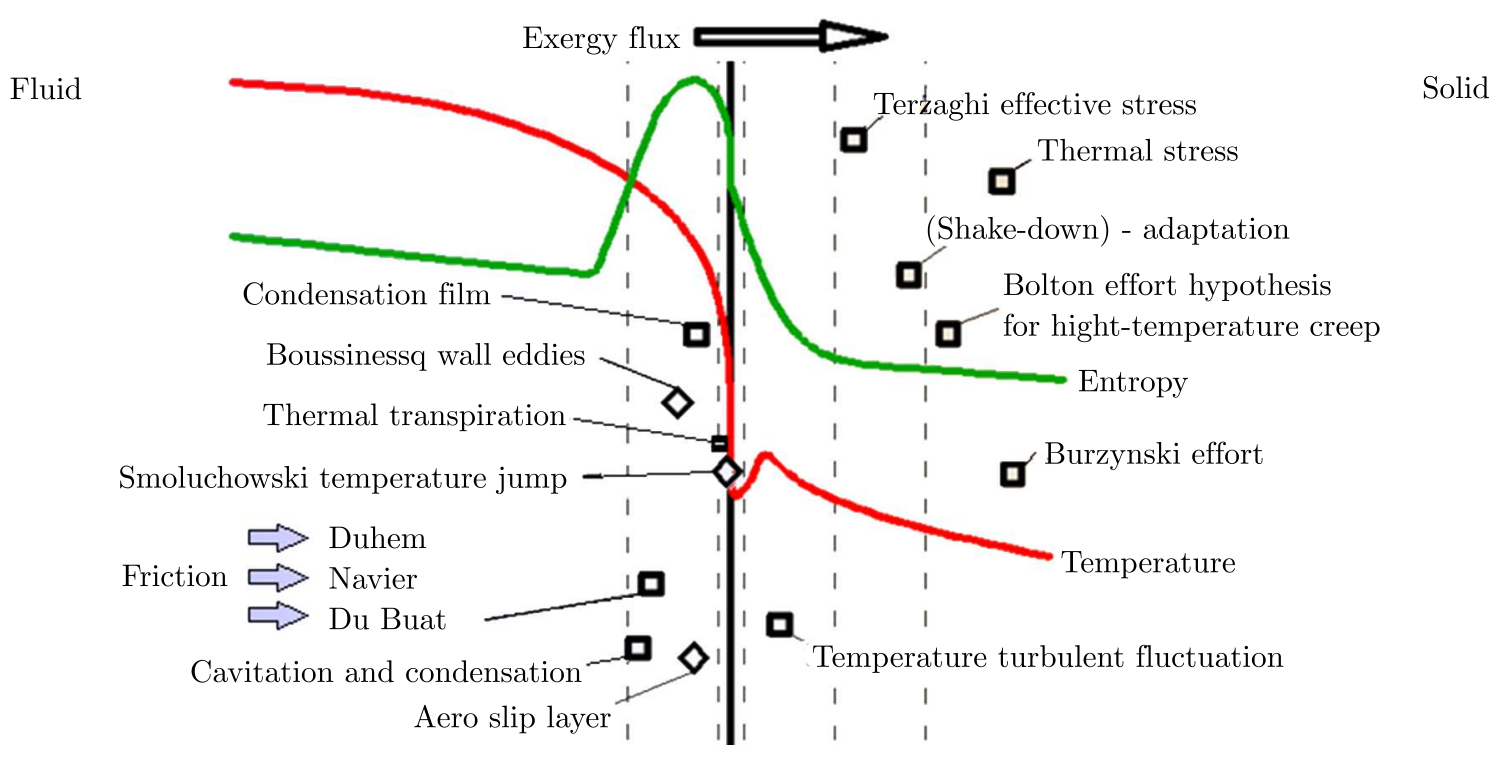

Fig. 1. Schematic presentation of emergencing asspects of mathemetical modelling that takes into acount fluid-solid interaction in the multiscale domain

\section{Collected effects of fluid-solid interaction in porous flow}

One example of applicability of this method to improve flow in porous media is a model of thermal transpiration described mainly via a mobility force. Beside the classical "bulk" behavior, wall stresses should also incline us to introduce new quantities such as surface friction force, surface mobility, etc. In the presented reasoning, it has been postulated that the generall surface "vis impressa" can be additively split into friction and mobility parts. Here, the mobility forces are defined as an ability of a fluid (gas or liquid) to flow along the wall without the assistance of, or even in opposition to, bulk (volume) forces. A whole description of this phenomenon is presented in (Badur et al., 2011, 2015). In this short communication, let us mention only reexamination of the Poiseuille-Knudsen-Reynolds equation in terms of a sum of three contributions: the bulk pressure driven flow and two mobility surface forces, mainly: the Knudsen surface slip driven flow and the Reynolds surface thermally driven flow.

A peculiar difficulty of the modeling of flow in porous media arises often when there is a need of applying some extension of Darcy equation, like for instance, the Brinkmann-Darcy-Forchheimer equation (Hooman, 2008). Treating that a laboratory nanopipe is fully equivalent to a single porous channel and looking for common effects of the bulk and surface motion, one may consider the following momentum flux integral in any cross section of a porous medium oriented by the tangential component of the unit vector $\mathbf{n}_{\tan }$

$$
\iint_{\text {bulk section }}(\rho \mathbf{v} \otimes \mathbf{v}+\mathbf{p}) \mathbf{n}_{\tan }+\oint_{\mathcal{C}}\left(\rho_{s} \mathbf{v}_{s} \otimes \mathbf{v}_{s}+\mathbf{p}_{s}+\mathbf{n}_{\text {nor }} \mathbf{p}+\mathbf{f}_{\partial V}\right) \mathbf{n}_{\tan } d \mathcal{C}=0
$$


In the above, $\rho \mathbf{v}$ and $\rho_{s} \mathbf{v}_{s}$ are the bulk and the surface momentum density vectors, $\rho$ and $\rho_{s}$ are the gas density in the bulk and on the boundary. Next, $\mathbf{p}=p_{i j} \mathbf{e}_{i} \otimes \mathbf{e}_{j}=\mathbf{p}^{\mathrm{T}}$ and $\mathbf{p}_{s}=\mathbf{p}_{s}^{\mathrm{T}}$ are the bulk and the surface flux momentum. The boundary force can be separated into surface friction and surface mobility, thus: $\mathbf{f}_{\partial V}=\mathbf{f}_{r}+\mathbf{f}_{m}$ (Badur et al., 2011, 2015). The total momentum influx will be

$$
\mathcal{M}=\iint_{\text {bulk section }} \rho \mathbf{v} v_{\tan } d A+\oint_{\mathcal{C}} \rho_{s} \mathbf{v}_{s} v_{s \tan } d \mathcal{C} \quad \mathcal{M}=\dot{m} \mathbf{v}_{r}
$$

where $\iint_{\text {bulk section }} \rho \mathbf{v} v_{\tan } d A$ - contribution from the bulk velocity and $\oint_{\mathcal{C}} \rho_{s} \mathbf{v}_{s} v_{s \tan } d \mathcal{C}$ - contribution from the slip velocity $v_{s \tan }=\mathbf{v}_{s} \mathbf{n}_{\tan }$. Using some arguments from technique of homogenization, we can postulate the existence of a resultant velocity $\mathbf{v}_{r}$ which is parallel to the vector of total momentum $(2.2)_{2}$. This vector is located somewhere in a geometrical center of the velocity profile. In many cases, independently of the shape of the cross section, the bulk profile of velocity is nearly flat and ending with the value of $v_{s \tan } \approx\left|\mathbf{v}_{s}\right|$ - the magnitude of slip velocity. In the above, according with the traditional Reynolds notation, $\dot{m}$ denotes the resultant mass flow rate. Taking into account that $\mathbf{f}_{\partial V}=\mathbf{f}_{r}+\mathbf{f}_{m}=\nu \mathbf{v}_{s}-c_{m \theta} \operatorname{grad}{ }_{s} \theta_{s}$, integral (2.1) can be reorganized, expressing explicitly the thermal mobility part $c_{m} \theta \operatorname{grad}{ }_{s} \theta_{s}$ with the thermomobility coefficient $c_{m} \theta$ and the slip friction part $\nu \mathbf{v}_{s}$, where $\nu$ is the Navier surface friction coefficient

$$
\dot{m} \mathbf{v}_{r}=\iint_{\text {Poiseuille }}(p \mathbf{I}-2 \mu \mathbf{d}) \mathbf{n} d A-\oint_{\text {Darcy }} \nu \mathbf{v}_{s} \mathbf{I}_{s} d \mathcal{C}-\oint_{\text {Reynolds }} c_{m \theta} \operatorname{grad}_{s}\left(\theta_{s}\right) \mathbf{I}_{s} d \mathcal{C}
$$

Since the porosity in any cross section of porous media is quite arbitrary, then the known procedure of homogenization can be applied, thus Eq. (2.3) leads finally to a 3D resultant equation (Vignoles et al., 2008)

$$
\mathbf{v}_{r}=-\left(\frac{P}{\mu} \mathbf{B}+\mathbf{D}_{K}\right) \frac{\operatorname{grad} P}{P}+\mathbf{D} \frac{\operatorname{grad} T}{T}
$$

where $\mathbf{v}_{r}$ is the resultant filtration velocity, $\mu$ - gas viscosity, $\mathbf{B}-$ permeability tensor, $\mathbf{D}_{K}-$ Knudsen accommodation diffusion tensor and $\mathbf{D}$ - thermal transpiration coefficient tensor. Here, the single surface pressure tensor $\mathbf{p}_{s}$ does not appear, slip velocity $\mathbf{v}_{s}$ or surface temperature $\theta_{s}$ either, since after homogenization their role retakes the capillarity pressure $P$ and the capillarity temperature $T$. Also, the two-dimensional surface $\operatorname{gradient} \operatorname{grad}_{s}(\cdot)$, due to homogenization, turns into the three-dimensional gradient $\operatorname{grad}(\cdot)$. This phenomenological model is based on the already averaged equations for the bulk flow resistance and the surface mobility forces, which means that some local coefficients, like the Navier slip and Reynolds transpiration can be used to a direct definition of $\mathbf{B}, \mathbf{D}_{K}$ and $\mathbf{D}$, respectively.

\section{References}

1. Badur J., Karcz M. Lemański M., 2011, On the mass and momentum transport in the Navier-Stokes slip layer, Microfluidics and Nanofluidics, 11, 439-449

2. BAdur J., ZióŁkowski P.J., ZióŁkowski P., 2015, On the angular velocity slip in nano flows, Microfluidics and Nanofluidics, 19, 191-198

3. Banaszkiewicz M., 2015, Multilevel approach to lifetime assessment of steam turbines, International Journal of Fatigue, 73, 39-47

4. Banaszkiewicz M., Rehmus-Forc A., 2015, Stress corrosion cracking of a $60 \mathrm{MW}$ steam turbine rotor, Engineering Failure Analysis, 51, 55-68 
5. Henry C., Minier J.-P., 2014, Progress in particle resuspension from rough surfaces by turbulent flows, Progress in Energy and Combustion Science, 45, 1-53

6. Hooman K., 2008, Heat and fluid flow in a rectangular microchannel filled with a porous medium, International Journal of Heat and Fluid Flow, 51, 5804-5810

7. Kowalewski T.A., Nakielski P., Pierini F., Zembrzycki K., PawŁowska S., 2016, Micro and nano fluid mechanics, [In:] Advances in Mechanics: Theoretical, Computational and Interdisciplinary Issues, M. Kleiber et al. (Eds.), 27-34

8. Kucaba-Piętal A., Walenta Z., Peradzyński Z., 2009, Molecular dynamics computer simulation of water flows in nanochannels, Bulletin of the Polish Academy of Sciences Technical Sciences, 57, 55-61

9. Lemański M., Karcz M., 2008, Performance of lignite-syngas operated tubular Solid Oxide Fuel Cell, Chemical and Process Engineering, 29, 233-248

10. Lewandowski T., Ochrymiuk T., Czerwińska J., 2011, Modeling of heat transfer in microchannel gas flow, ASME Journal of Heat Transfer, 133, 022401-1

11. Morini G.L., Yang Y., Chalabi H., Lorenzini M., 2011, A critical review of the measurement techniques for the analysis of gas microflow through microchannels, Experimental Thermal and Fluid Science, 35, 849-893

12. Nakielski P., PawŁowska S., Pierini F., Liwińska W., Hejduk P., Zembrzycki K., Zabost E., Kowalewski T., 2015, Hydrogel nanofilaments via core-shell electrospinning, PLoS ONE, 10, 6, e0129816, DOI: 10.1371/journal.pone.0129816

13. Nitoń P., Żywociński A., FiaŁkowski M., HoŁyst R., 2013, A “nano-windmill” driven by a flux of water vapour: a comparison to the rotating ATPase, Nanoscale, 5, 9732-9738

14. O’Hare L., Lockerby D.A., Reese J.M., Emerson D.R., 2007, Near-wall effects in rarefied gas micro-flows: some modern hydrodynamic approaches, International Journal of Heat and Fluid Flow, 28, 37-43

15. Pęcherski R.B., Szeptyński P., Nowak M., 2011, An extension of Burzyński hypothesis of material effort accounting for the third invariant of stress tensor, Archives of Metalurgy and Materials, 56, 2, 503-508

16. Reese M., Gallis M.A., Lockerby D.A., 2003, New directions in fluid dynamics: non-equilibrium aerodynamic and microsystem flows, Philosophical Transactions A: Mathematical, Physical and Engineering Sciences, 361, 2967-2988

17. Thomson P.A., Trojan S.M., 1997, A general boundary condition for liquid flow at solid surface, Nature, 389, 360-362

18. Vadillo G., Fernandez-Saez J., Pęcherski R.B., 2011, Some applications of Burzynski yield condition in metal plasticity, Materials and Design, 32, 628-635

19. Vignoles G.L., Charrier P., Preux C., Dubroca B., 2008, Rarefied pure gas transport in non-isothermal porous media: effective transport properties from homogenization of the kinetic equation, Transport in Porous Media, 73, 2, 211-232

20. ZıóŁkowski P., BAdur J., 2014, Navier number and transition to turbulence, Journal of Physics: Conference Series, 530, 012035, DOI: 10.1088/1742-6596/530/1/012035 